JOURNAL OF INTEGRAL EQUATIONS AND APPLICATIONS

Volume 13, Number 3, Fall 2001

\title{
ON THE ESSENTIAL NORM OF THE DOUBLE LAYER POTENTIAL ON POLYHEDRAL DOMAINS AND THE STABILITY OF THE COLLOCATION METHOD
}

\author{
OLAF HANSEN
}

\begin{abstract}
In this article we study the double layer operator on a polyhedral domain $D$ in $\mathbf{R}^{3}$. We construct weighted norms for a class of nonconvex cones, such that the double layer operator has a norm smaller than $1 / 2$ with respect to this weighted norm. Applied to the rectangular example of Král and Wendland, our method reproduces their weight function. For certain domains we are able to extend this local weighted norm to the whole boundary $S$ of $D$ in such a way that the double layer operator has an essential norm smaller than $1 / 2$ on $S$. This allows us to prove stability for the collocation method with piecewise polynomial trial functions of arbitrary degree if we introduce some modification near the vertices and if the grid respects the weighted norm in some given sense.
\end{abstract}

1. Introduction. In this paper we consider polyhedral domains $D \subset \mathbf{R}^{3}$ with boundary $S=\partial D$. In the space $\mathcal{C}(S)$ we study the equation

$$
\left(\frac{1}{2} I-\bar{W}\right) u=f, \quad f \in \mathcal{C}(S),
$$

where the double layer operator $\bar{W}$ is defined by

$$
(\bar{W} u)(x)=W u(x)+\left(\frac{1}{2}-d(x)\right) u(x), \quad x \in S,
$$

with

$$
d(x):=\lim _{r \searrow 0} \frac{\operatorname{volume}\left(B_{r}(x) \cap D\right)}{\operatorname{volume}\left(B_{r}(x)\right)}
$$

Key words and phrases. Double layer potential, polyhedral domains, collocation method, stability.

1991 Mathematics Subject Classification. 65R20, 65N12, 35J25, 45B05.

The article was written while the author was visiting the University of Iowa, Iowa City.

Received by the editors on November 6, 2000, and in revised form on March 21, 2001.

Copyright (C)2001 Rocky Mountain Mathematics Consortium 
and

$$
(W u)(x):=\frac{1}{4 \pi} \int_{S} \frac{n(y) \cdot(y-x)}{\|x-y\|^{3}} u(y) d y,
$$

see $[\mathbf{1}],[\mathbf{1 1}],[\mathbf{1 2}],[\mathbf{1 3}]$. For the following we denote the kernel of the above integral operator by $k$

$$
k(x, y):=\frac{1}{4 \pi} \frac{n(y) \cdot(y-x)}{\|x-y\|^{3}}, \quad y \in S, x \in \mathbf{R}^{3} \backslash\{y\} .
$$

The kernel $k$ is well defined if $y$ does not lie on an edge. If $y$ lies on an edge, we define $k(x, y)=0, x \in S$. Equation (1.1) is well studied, and it is used, for example, in applications for the solution of the Dirichlet problem, see $[\mathbf{2}],[\mathbf{4}]$,

$$
\left\{\begin{aligned}
\Delta v(x) & =0 & & x \in D \\
v(x) & =f(x) & & x \in S
\end{aligned}\right.
$$

Despite the fact that (1.1) has been studied for a long time, some properties of the operator $\bar{W}$ are still not clear for the case of polyhedral domains. Rathsfeld proved in his article [14, Theorem 0.1, Corollary 0.2 ] that the spectral radius in the space of continuous functions is smaller than $1 / 2$ on polyhedral domains, and he proved the invertibility of equation (1.1). Further results on the invertibility can be found in [9], [10]. Here we will prove that the essential norm, see (1.7), of the double layer operator is smaller than $1 / 2$ on certain polyhedral domains, see Theorem 3.1 for a precise description. This result is not included in the above mentioned articles, and this result is particularly useful for the proof of the stability of the collocation method, see Section 4.

One of the important results in the above-cited papers which is important for second kind integral equations like (1.1) is the Fredholm property of these operators. In the case of nonsmooth boundaries $S$, the operator $\bar{W}$ is not compact, and so it is not clear if the Fredholm alternative holds for equation (1.1). A sufficient condition would be

$$
\begin{aligned}
\|\bar{W}\|_{\mathcal{C}(S), \text { ess }} & :=\inf \left\{\|\bar{W}-T\|_{\mathcal{C}(S)} \mid T \in \mathcal{L}(\mathcal{C}(S), \mathcal{C}(S)), T \text { compact }\right\}, \\
& <\frac{1}{2}
\end{aligned}
$$




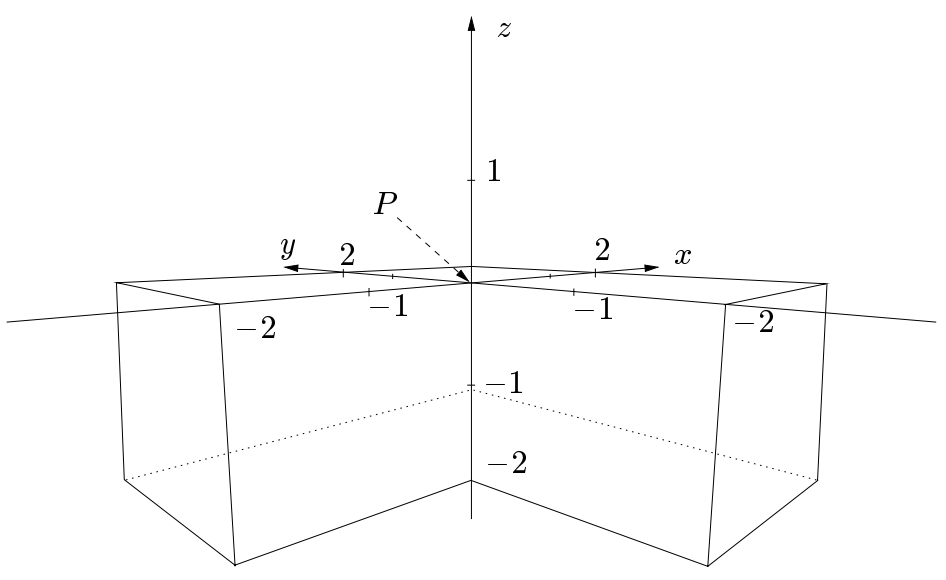

FIGURE 1. An example of a domain $D$ with $\|\bar{W}\|_{\mathcal{C}\left(S_{1}\right), \text { ess }}>0.5$.

But Král and Wendland [12] have shown that, even in the case of the simple domain $D_{1}$, with boundary $S_{1}$ (see Figure 1 ), the condition (1.8) is not fulfilled.

To study this it is sufficient to consider the operator $\bar{W}$ only locally around $P$. For this localized consideration we introduce the infinite cone $K_{P}$

$$
K_{P}:=\left\{P+\lambda(x-P) \mid x \in B_{\varepsilon}(P) \cap S, \lambda \geq 0\right\},
$$

$\varepsilon>0$ sufficiently small. We will always assume that $P=0$ holds and define the spherical polygon $\gamma_{P}$ by

$$
\gamma_{P}:=K_{P} \cap B_{1}(0) .
$$

To substitute for the lack of (1.7)-(1.8), Král and Wendland introduced a weighted norm on $\mathcal{C}\left(S_{1}\right)$ which is compatible with the usual supremum norm. If $w \in L^{\infty}(S)$ denotes a function with $0<c<w(x)<C, x \in S$, almost everywhere, the norm

$$
\|u\|_{\mathcal{C}(S), w}:=\sup \left\{\frac{|u(x)|}{w(x)} \mid x \in S\right\}
$$


is equivalent to $\|\cdot\|_{\mathcal{C}(S)}$, and the set of compact operators does not change.

How does the weight function of Král and Wendland look near the vertex $P$, respectively on the cone $K_{P}$ ? In their situation we have the picture given in Figure 2. The spherical polygon $\gamma=\cup_{j=1}^{5} \gamma_{j}$ is partitioned according to the above picture and the cone $K_{P}$ is partitioned in a similar way

$$
\begin{aligned}
K_{P} & =\bigcup_{j=1}^{5} K_{j} \\
& =\bigcup_{j=1}^{5}\left\{\lambda x \mid \lambda \geq 0, x \in \gamma_{j}\right\} .
\end{aligned}
$$

The weight function of Král and Wendland is constant on every $K_{j}$,

$$
w(x)=\left\{\begin{array}{rl}
1 & x \in K_{4} \cup K_{5}, \\
\approx 0.783612 & x \in K_{1} \cup K_{3}, \\
\approx 0.261204, & x \in K_{2} .
\end{array}\right.
$$

This weight function is found by some combinatoric estimates and the solution of a quadratic equation. The result is

$$
\|\bar{W}\|_{\mathcal{C}\left(K_{P}\right), w} \lesssim 0.478553 .
$$

This result proves (1.8) and can also be used as a starting point for the stability analysis of the collocation method. This connection to the stability of the collocation method makes the study of the essential norm of the operator $\bar{W}$ especially interesting.

In Section 2 we use the above ideas as a starting point for finding suitable weights for a larger class of spherical cones. Given a cone $K$, the generating spherical polygon $\gamma_{P}$ and a partition $\mathcal{P}\left(\gamma_{P}\right)=$ $\left\{\gamma_{1}, \ldots, \gamma_{n}\right\}$ of $\gamma_{P}$, we will construct a matrix $M=M\left(\mathcal{P}\left(\gamma_{P}\right)\right) \in \mathbf{R}^{n, n}$. The maximal eigenvalue $\alpha$ is always positive, and if this eigenvalue is smaller than $1 / 2$, we can construct a weight function $w$ such that

$$
\|\bar{W}\|_{\mathcal{C}\left(K_{p}\right), w} \leq \alpha .
$$

We are also able to show that this weight function is unique for this partition in a particular sense. This result already gives the invertibility 


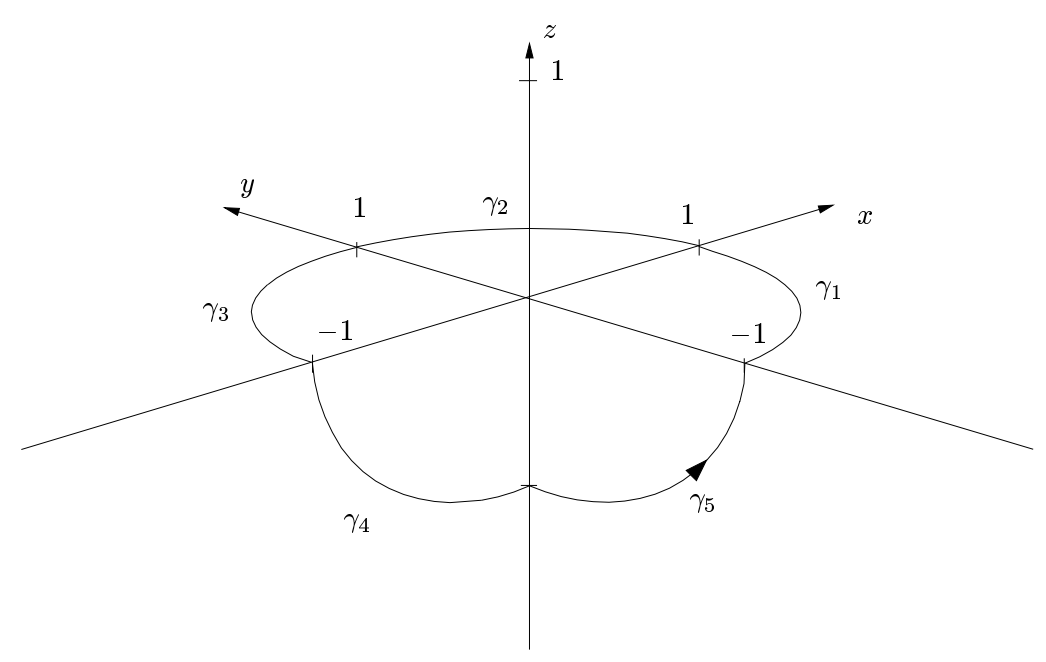

FIGURE 2. The spherical polygon $\gamma$ and its partition.

of the operator $1 / 2 I-\bar{W}$ on suitable cones, see Definition 2.3. But if we want to estimate the essential norm of the double layer operator on $S$ we have to glue together the local weighted norms. The corresponding sufficient condition (2.18) is given in Definition 2.3.

The above condition enables us in Section 3 to construct a weighted norm on $S$ such that the essential norm of the double layer operator $\bar{W}$ on $S$ is smaller than $1 / 2$ with respect to this norm. The splitting of the operator $\bar{W}=\bar{W}_{\varepsilon}+\bar{K}_{\varepsilon}$, where $\bar{W}_{\varepsilon}$ has a norm smaller than $1 / 2$ and a compact operator $\bar{K}_{\varepsilon}$, is so explicit that we can use this splitting in Section 4 to prove the stability of the collocation method. Here we allow an arbitrary degree for the trial functions, but we must use some $i^{*}$ modification, see [5], near the vertices (it will be called $\varepsilon^{*}$ modification) to guarantee the stability. The triangulations we consider have to respect the weighted norm in some sense, see (4.13), but otherwise they can be chosen arbitrarily.

2. On polyhedral cones and their corresponding matrices. In this section we consider an arbitrary infinite polygonal cone $K$, the spherical polygon $\gamma=K \cap B_{1}(0)$ and a given partition $\mathcal{P}(\gamma)=$ $\left\{\gamma_{1}, \ldots, \gamma_{n}\right\}$ of $\gamma$, where every $\gamma_{j}, j=1(1) n$ should lie in a plane. 
Connected to the partition of $\gamma$ is a partition $K=\cup_{j=1}^{n} K_{j}$, according to equation (1.13). We are looking for a weight function $w: K \rightarrow \mathbf{R}$,

$$
w(x)=\omega_{j}>0, \quad x \in K_{j}
$$

such that

$$
\|\bar{W}\|_{\mathcal{C}(S), w}<\frac{1}{2}
$$

First we consider a sector $\Delta\left(\varphi_{0}\right) \subset \mathbf{R}^{3}, \varphi_{0} \in(0, \pi)$, defined by

$$
\Delta\left(\varphi_{0}\right)=\left\{r\left(\begin{array}{c}
\cos (\varphi) \\
\sin (\varphi) \\
0
\end{array}\right):|\varphi| \leq \varphi_{0}, r \geq 0\right\} .
$$

For $x \in \mathbf{R}^{n} \backslash \Delta\left(\varphi_{0}\right)$ we define

$$
W_{E, \varphi_{0}}(x):=\int_{\Delta\left(\varphi_{0}\right)}|k(x, y)| d y, \quad x \in \mathbf{R}^{3} \backslash \Delta\left(\varphi_{0}\right),
$$

the integral of the absolute value of the kernel of the double-layer potential. A scaling argument gives

$$
W_{E, \varphi_{0}}(x)=W_{E, \varphi_{0}}\left(\frac{x}{\|x\|}\right)
$$

and therefore it is enough to consider

$$
W_{E, \varphi_{0}}: S^{2} \backslash \Delta\left(\varphi_{0}\right) \longrightarrow \mathbf{R} .
$$

Here we recall the fact that $W_{E, \varphi_{0}}$ is nothing other than the integral of the absolute value of the double layer potential with constant layer function for the sector $\Delta\left(\varphi_{0}\right)$. This also implies that $W_{E, \varphi_{0}}(x)$ is equal to the normalized solid angle of $\Delta\left(\varphi_{0}\right)$ seen from $x$. So it is natural to extend $W_{E, \varphi_{0}}$ to $S^{2}$ by

$$
W_{E, \varphi_{0}}(x):=\frac{1}{2}, \quad x \in S^{2} \cap \Delta\left(\varphi_{0}\right),
$$




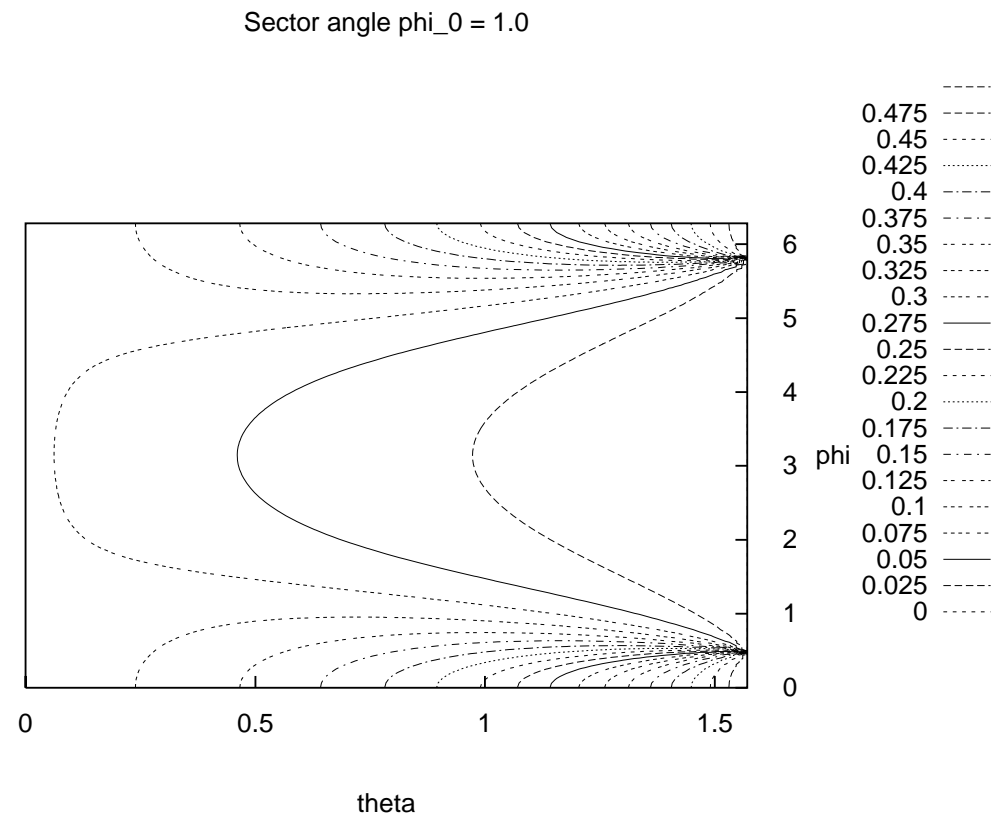

FIGURE 3. The contour of $W_{E, 1.0}$ on $S^{+} \cong[0, \pi / 2] \times[0,2 \pi]$.

because the (normalized) solid angle approaches $1 / 2$ if one comes closer and closer to the sector $\Delta\left(\varphi_{0}\right)$, see also [13]. Then $W_{E, \varphi_{0}}$ is continuous (jump relation of the double layer potential) in

$$
S^{2} \backslash\left\{\left(\cos \left(\varphi_{0}\right),-\sin \left(\varphi_{0}\right), 0\right)^{T},\left(\cos \left(\varphi_{0}\right), \sin \left(\varphi_{0}\right), 0\right)^{T}\right\} .
$$

The value of $W_{E, \varphi_{0}}$ is equal to

$$
W_{E, \varphi_{0}}(\theta, \varphi)=\frac{1}{4 \pi} \int_{-\varphi_{0} / 2}^{\varphi_{0} / 2} \frac{|\cos (\theta)|}{1-\sin (\theta) \cos (\tau-\varphi)} d \tau
$$

which follows by an easy calculation. Here we used the coordinates $(\theta, \varphi) \in[0, \pi] \times[0,2 \pi)$ for $S^{2}$ given by $(\theta, \varphi) \rightarrow(\sin (\theta) \cos (\varphi)$, $\sin (\theta) \sin (\varphi), \cos (\theta))^{T}$. Using these coordinates we get, for example, Figures 3 and 4 of $W_{E, \varphi_{0}}$. The contour lines are for constant $W_{E, \varphi_{0}}(\theta, \varphi)$.

For every $j \in\{1, \ldots, n\}$ we denote by $R_{j}$ a rotation which maps $K_{j}$ bijectively onto $\Delta\left(\varphi_{j}\right), \varphi_{j} \in(0, \pi)$; here we recall the fact that the 


\section{Sector angle phi $\_=4.0$}

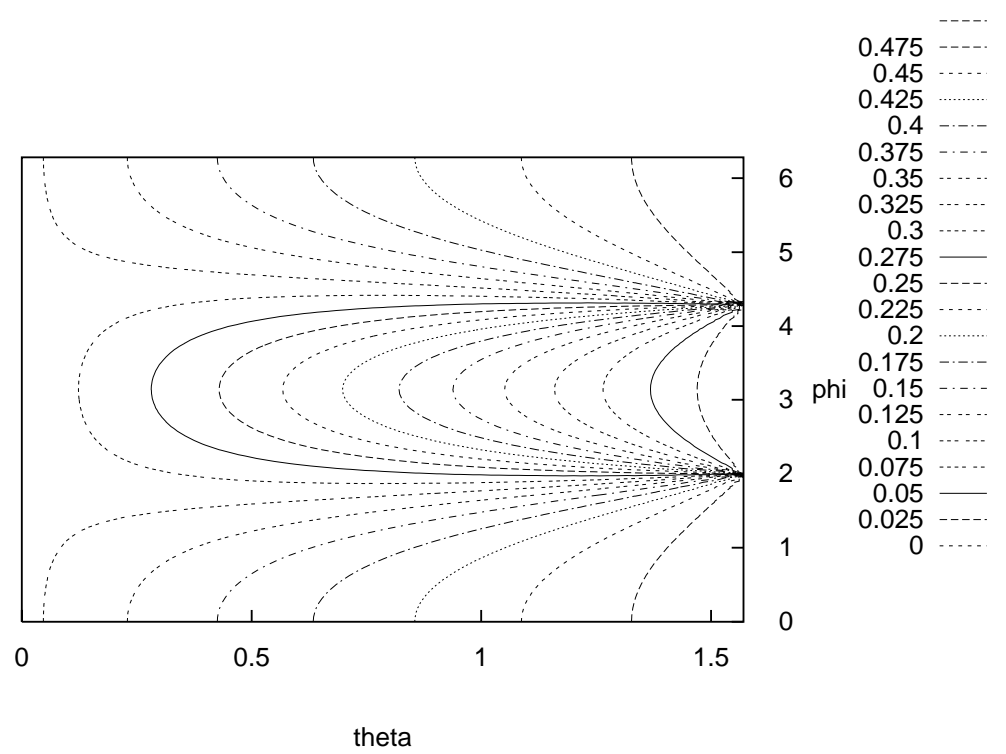

FIGURE 4. The contour of $W_{E, 4.0}$ on $S^{+} \cong[0, \pi / 2] \times[0,2 \pi]$.

vertex of $K$ is at zero. The matrix $M=M(\mathcal{P}(\gamma)):=\left(m_{i, j}\right)_{i, j=1(1) n}$ is defined by

$$
m_{i, j}:= \begin{cases}\sup \left\{W_{E, \varphi_{j}}(x) \mid x \in R_{j} K_{i} \backslash \Delta\left(\varphi_{j}\right)\right\}, & i \neq j \\ 0, & \text { otherwise. }\end{cases}
$$

All entries of $M$ (if possible we suppress the dependence on the partition) outside the diagonal are greater than zero, and the theorem of Perron and Frobenius proves the existence of a one-dimensional subspace $\langle\vec{\omega}\rangle$ in $\mathbf{R}^{n}$, such that

$$
\left\{\begin{aligned}
\vec{\omega}= & \left(\omega_{1}, \ldots, \omega_{n}\right), \omega_{i}>0, \\
& \text { and } \\
M \vec{\omega}= & \rho(M) \vec{\omega}, \rho(\omega)>0 .
\end{aligned}\right.
$$

In the following we will always assume that the weight function $w$ is defined by (2.1), $\omega_{j}, j=1(1) n$, given by (2.9), (2.10) and $\max \left\{\omega_{j} \mid j=\right.$ $1(1) n\}=1$. 
Lemma 2.1. Given a cone $K$ with a partition $K=\cup_{j=1}^{n} K_{j}$ corresponding to the partition $\mathcal{P}(\gamma)=\left\{\gamma_{1}, \ldots, \gamma_{n}\right\}$ of the spherical polygon $\gamma$, we get

$$
\|\bar{W}\|_{\mathcal{C}(K), w} \leq \rho(M(\mathcal{P}(\gamma))),
$$

where the weight function $w$ on $K$ is given by (2.1), (2.9) and (2.10).

Proof. Let $u \in L^{\infty}(K),\|u\|_{L^{\infty}(K), w} \leq 1$ and $x \in K_{i}$. We can assume $x \notin K_{j}, i \neq j$. Then we have

$$
\begin{aligned}
&|(\bar{W} u)(x)| \leq \sum_{j=1, i \neq j}^{n} \int_{K_{j}}|k(x, y)||u(y)| d y \\
& \leq \sum_{j=1, i \neq j}^{n} \omega_{j} \int_{K_{j}}|k(x, y)| d y \\
&=\sum_{j=1, i \neq j}^{n} \omega_{j} \int_{R_{j} K_{j}}\left|k\left(R_{j} x, y\right)\right| d y \\
&=\sum_{j=1, i \neq j}^{n} \omega_{j} \int_{\Delta\left(\varphi_{j}\right)}\left|k\left(R_{j} x, y\right)\right| d y \\
& \stackrel{(2.9)}{\leq} \sum_{j=1, i \neq j}^{n} \omega_{j} m_{i, j} \\
& \stackrel{(2.10)}{=} \rho(M) \omega_{i} .
\end{aligned}
$$

Note, $k(x, y)=0$ for $x, y \in K_{i}$.

Remark. After reading the proof of Lemma 2.1, one immediately asks if there are other vectors $\vec{\nu}=\left(\nu_{1}, \ldots, \nu_{n}\right)^{T}, \nu_{i}>0, i=1(1) n$, such that

$$
M \vec{\nu} \leq \alpha \vec{\nu}, \quad \alpha \in(0, \rho(M)) .
$$

This would imply that we can reduce the essential norm of $\bar{W}$ by choosing the $\nu_{i}$ instead of the $\omega_{i}$ as our weight function. But here we have the result of Woodbury [15, Theorem 1] which states that if $M$ is positive, $\vec{\nu} \geq 0, \lambda \in \mathbf{R}$ and

$$
M \vec{\nu} \leq \lambda \vec{\nu},
$$


then we get $\lambda \geq \rho(M)$. This shows that the choice of the eigenvector $\vec{\omega}$ is optimal in this sense.

First we take a look at the example of Král and Wendland in our context. We denote by $K_{K W}$ the corresponding cone. The partition of $\gamma_{K W}$ is then given by

$$
\gamma_{K W}=\left\{\widetilde{\gamma}_{1}, \widetilde{\gamma}_{2}, \widetilde{\gamma}_{3}\right\}, \quad \tilde{\gamma}_{1}:=\gamma_{1} \cup \gamma_{3}, \quad \tilde{\gamma}_{2}:=\gamma_{2}, \quad \widetilde{\gamma}_{3}:=\gamma_{4} \cup \gamma_{5},
$$

see Figure 2. We remark here that this partition does not fit into our general scheme (2.9), but it is easy to extend our scheme to this special partition, but now we get a nonzero diagonal element, because $\widetilde{\gamma}_{3}$ does not lie in a plane. The matrix $M_{K W}$ is given by

$$
M_{K W}=\left(\begin{array}{ccc}
0 & 0 & 0.375 \\
0 & 0 & 0.125 \\
0.250 & 0.125 & 0.250
\end{array}\right)
$$

the spectral radius is given by $\rho\left(M_{K W}\right) \approx 0.478553$ and the corresponding eigenvector $\vec{\omega}_{K W}$ corresponds exactly to the weights in equation (1.14). So our method works after a slight extension to nonplane partitions. But the question is if one can find suitable partitions for a larger class of cones. We propose the following.

Given a cone $K$ with a starting partition of plane sectors $K_{j}, j=$ $1(1) n$, we define a partition $\mathcal{P}^{N}(\gamma)$ by dividing each $\gamma_{j}, j=1(1) n$, into $N$ equal plane sectors $\gamma_{j, l}^{N}, l=1(1) N$. Correspondingly we have $K=\cup_{j=1}^{n} \cup_{l=1}^{N} K_{j, l}^{N}$. We will also denote the corresponding matrix with an upper index $N$.

In addition to the Král-Wendland example with 5 starting partitions (see Figure 2), we consider the nonconvex cone $K_{A}$ with a spherical polygon $\gamma_{A}$ defined by the corners $\left(0^{\circ}, 0^{\circ}\right),\left(90^{\circ}, 0^{\circ}\right),\left(80^{\circ}, 45^{\circ}\right)$ and $\left(90^{\circ}, 90^{\circ}\right)$ on the unit sphere. The spherical polygon evidently has a partition with 4 plane sections. Now we can calculate with a program the matrices $M_{K W}^{N}$, respectively $M_{A}^{N}$, for a sequence of $N$ values, and as soon as we get $\rho\left(M_{K W}^{N}\right)<0.5$, respectively $\rho\left(M_{A}^{N}\right)<0.5$, we can stop; then we know that there exists a suitable weight function such that (2.2) is fulfilled. We get the results shown in Figure 5 .

These results show that by our very simple strategy we find that both cones have a weight function for which the essential norm of $\bar{W}$ 


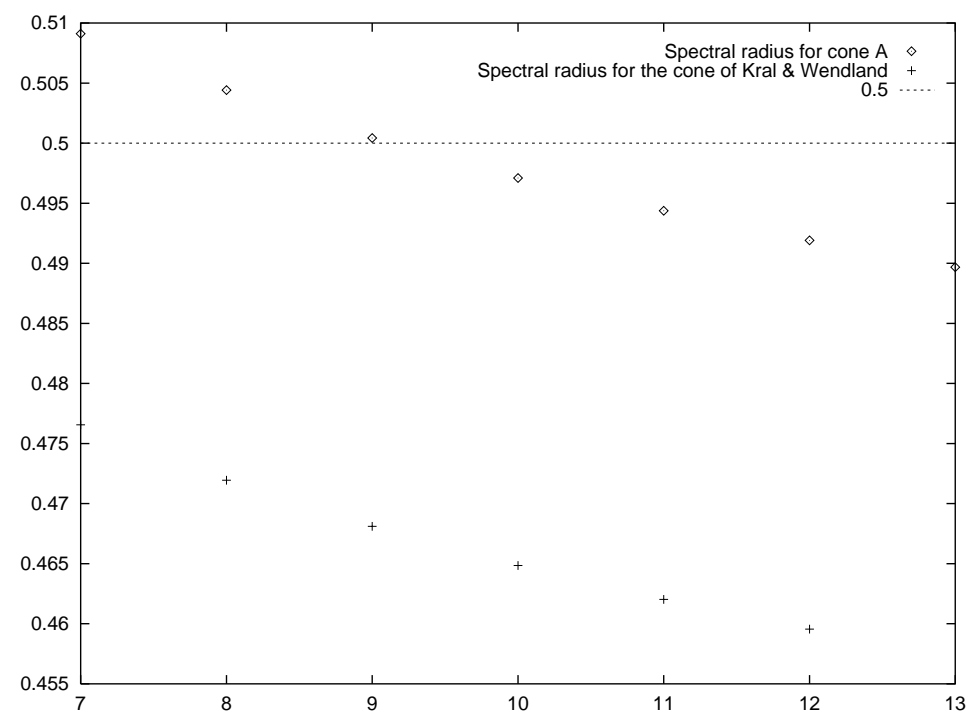

FIGURE 5. The spectral radii for different values of $N$ and the two cones $K_{K W}$ and $K_{A}$.

is smaller than 0.5. In their paper [12] Král and Wendland consider a further rectangular cone; here also our method works and we find suitable weights. The cone $K_{A}$ is only 'a little bit' nonconvex. What happens if one considers more nonconvex cones? We denote by $K_{B}$ the cone whose spherical polygon is given by the corners $\left(0^{\circ}, 0^{\circ}\right),\left(90^{\circ}, 0^{\circ}\right)$, $\left(45^{\circ}, 45^{\circ}\right)$ and $\left(90^{\circ}, 90^{\circ}\right)$ on the unit sphere; see Figure 6 . Here we find no suitable weight function with fewer than 100 partitions of each of the faces of $K_{B}$, indicated in Figure 7 .

The above considerations naturally lead to the question of whether a function $w \in L^{\infty}(\gamma), 0<c \leq w(x) \leq C, x \in \gamma$, exists almost everywhere, and a $\lambda \in(0,1 / 2)$, such that

$$
\lambda w(x) \leq \int_{K}|k(x, y)| w(y) d y, \quad x \in \gamma .
$$

We remark that $w$ actually only depends on the norm of $x$. Then the operator norm of $\bar{W}$ with respect to $\|\cdot\|_{\mathcal{C}(K), w}$ would be smaller than 


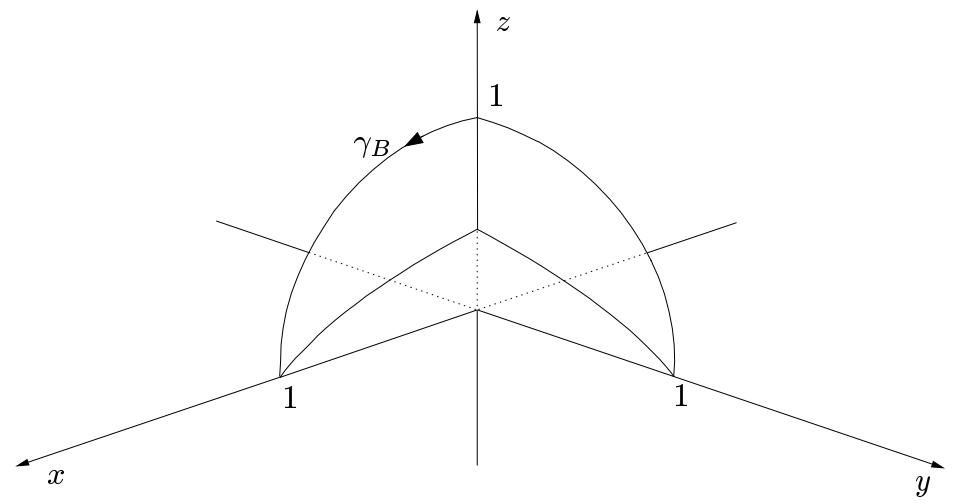

FIGURE 6. The cone $K_{B}$.

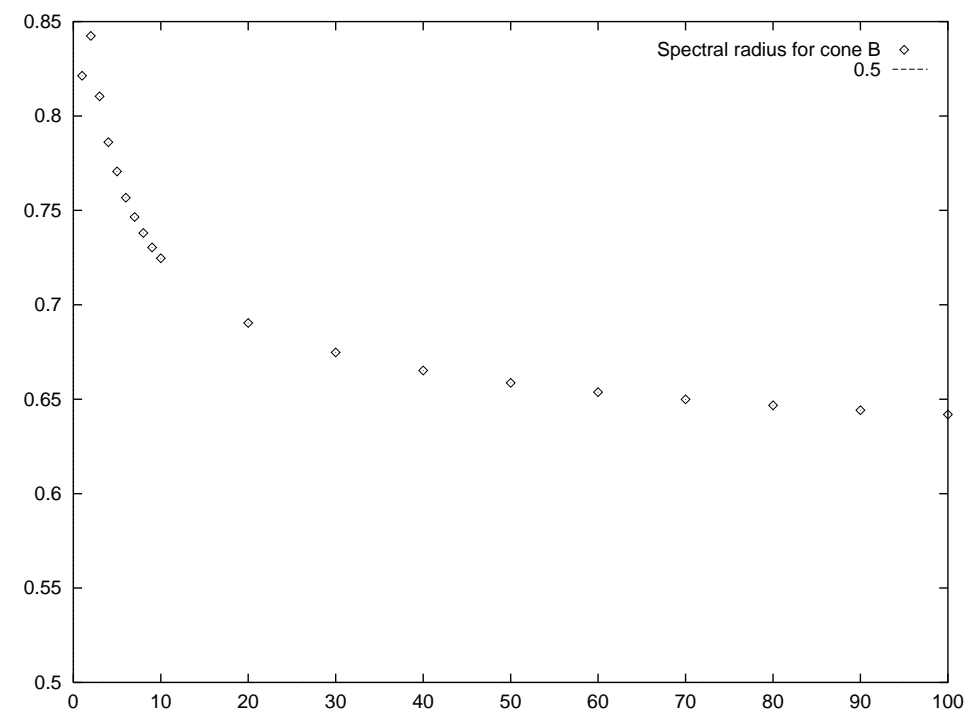

FIGURE 7. The spectral radii for $M_{B}^{N}$, with different $N$ values below 100 . 
$1 / 2$. Now we take a look at the operator on the righthand side of equation (2.12). We introduce the following notations $x=r q, q \in \gamma$, $r>0$.

$$
\begin{aligned}
\int_{K} \mid & k(x, y) \mid w(y) d y \\
= & \frac{1}{4 \pi} \int_{\gamma} \int_{0}^{\infty} \frac{|n(p) \cdot(r q-s p)|}{\|r q-s p\|^{3}} s w(p) d s d p \\
& =\frac{1}{4 \pi} \int_{\gamma} w(p) \int_{0}^{\infty} \frac{|n(p) \cdot(r q-s p)| s}{\left(r^{2}-2 r s(p, q)+s^{2}\right)^{3 / 2}} d s d p \\
& =\frac{1}{4 \pi} \int_{\gamma} \frac{w(p)}{r} \int_{0}^{\infty} \frac{|n(p) \cdot(q-(s / r) p)|}{\left(1-2(s / r)(p, q)+(s / r)^{2}\right)^{3 / 2}} \frac{s}{r} d s d p \\
& =\frac{1}{4 \pi} \int_{\gamma} w(p) \int_{0}^{\infty} \frac{|n(p) \cdot(q-\tau p)|}{\left(1-2 \tau(p, q)+\tau^{2}\right)^{3 / 2}} \tau d \tau d p \\
n(p) \cdot p=0 & \int_{\gamma} w(p)|n(p) \cdot q| \int_{0}^{\infty} \frac{\tau}{\left(1-2 \tau(p, q)+\tau^{2}\right)^{3 / 2}} d \tau d q \\
& =\frac{1}{4 \pi} \int_{\gamma} \frac{|n(p) \cdot q|}{1-(p, q)} w(p) d p \\
& =: \\
& (V w)(q) .
\end{aligned}
$$

With this integral operator with positive kernel $V$ on $\gamma$ we can reformulate (2.12) in the following way. Does a positive function $w \in L^{\infty}(\gamma)$ and a constant $\lambda \in(0,1 / 2)$ exist such that

$$
(V w)(x) \leq \lambda w(x), \quad x \in \gamma .
$$

The theorem of Woodbury suggests that $\lambda<\rho(V)$ is impossible, but this is not clear. If $V$ would be a compact operator the theorem of Krein and Rutman would imply the existence of a positive function $w$ such that

$$
V w=\rho(V) w,
$$

but the next lemma shows that the operator is not compact.

Lemma 2.2. Let $\gamma \subset S^{2}$ be a spherical polygon and $V$ be defined by (2.13). The operator $V$ is locally around each vertex $\xi$ of $\gamma$, isomorphic 
to a Mellin convolution operator $L_{\alpha}$ on a finite interval with kernel

$$
l_{\alpha}(x)=\frac{|\sin (\alpha)|}{2 \pi} \frac{x}{1+2 \cos (\alpha) x+x^{2}},
$$

where $\alpha \in(-\pi, \pi)$ is the angle of $\gamma$ at $\xi$.

Proof. Let $\xi \in \gamma$ be a vertex of $\gamma$. We can assume that $\xi=(0,0,1)^{T}$ and that we have a parametrization $\beta$ of $\gamma$ such that $\left.\beta\right|_{\left[-\delta_{0}, \delta_{0}\right]}, \delta_{0}>0$, is given by

$$
\begin{aligned}
& \beta(\tau)=\left(\begin{array}{c}
-\cos (\alpha) \sin (\tau) \\
-\sin (\alpha) \sin (\tau) \\
\cos (\tau)
\end{array}\right), \quad \tau \in\left[-\delta_{0}, 0\right], \\
& \beta(\tau)=\left(\begin{array}{c}
\sin (\tau) \\
0 \\
\cos (\tau)
\end{array}\right), \quad \tau \in\left[0, \delta_{0}\right] .
\end{aligned}
$$

For $\tau \in\left[-\delta_{0}, 0\right], w \in L^{\infty}\left(\left[-\delta_{0}, \delta_{0}\right]\right),(V w)(\tau)$ is given by

$$
\begin{aligned}
& (V w)(\tau) \\
& =\frac{1}{4 \pi} \int_{0}^{\delta_{0}} \frac{|\sin (\alpha)| \sin (-\tau) w(\nu)}{1-(-\cos (\alpha) \sin (\tau) \sin (\nu)+\cos (\tau) \cos (\nu))} d \nu \\
& =-\frac{|\sin (\alpha)|}{4 \pi} \int_{0}^{\delta_{0}}(2 \sin (\tau / 2) \cos (\tau / 2) w(\nu)) \\
& \quad \cdot\left(2 \cos (\tau / 2)^{2} \sin (\nu / 2)^{2}+2 \sin (\tau / 2)^{2} \cos (\nu / 2)^{2}\right. \\
& \quad+4 \cos (\alpha) \sin (\tau / 2) \cos (\tau / 2) \sin (\nu / 2) \cos (\nu / 2))^{-1} d \nu \\
& =-\frac{|\sin (\alpha)|}{4 \pi} \quad \int_{0}^{\delta_{0}} \frac{\tan (\tau / 2) w(\nu)}{\tan (\nu / 2)^{2}+\tan (\tau / 2)^{2}+2 \cos (\alpha) \tan (\nu / 2) \tan (\tau / 2)} \frac{d \nu}{\cos (\nu / 2)^{2}}
\end{aligned}
$$

Define the operator

$$
\begin{gathered}
T: L^{\infty}\left(\left[-\delta_{0}, \delta_{0}\right]\right) \longrightarrow L^{\infty}\left(\left[-\tan \left(\delta_{0} / 2\right), \tan \left(\delta_{0} / 2\right)\right]\right), \\
(T w)(\mu):=w(2 \arctan (\mu)),
\end{gathered}
$$


and the substitution

$$
\mu=\tan (\nu / 2)
$$

see [7]. So we get

$$
(V w)(\tau)=-\frac{|\sin (\alpha)|}{2 \pi} \int_{0}^{\delta_{0}} \frac{\tan (\tau / 2) w(2 \arctan (\mu))}{\mu^{2}+2 \cos (\alpha) \mu \tan (\tau / 2)+\tan (\tau / 2)^{2}} d \mu
$$

and therefore

$$
\begin{aligned}
(T V w)(\kappa) & =-\frac{|\sin (\alpha)|}{2 \pi} \int_{0}^{\delta_{0}} \frac{\kappa}{\mu^{2}+2 \cos (\alpha) \mu \kappa+\kappa^{2}}(T w)(\kappa) d \mu \\
& =-\int_{0}^{\delta_{0}} l_{\alpha}\left(\frac{\kappa}{\mu}\right)(T w)(\mu) \frac{d \mu}{\mu} .
\end{aligned}
$$

This finally implies

$$
\left.V\right|_{\left[-\delta_{0}, \delta_{0}\right]}=T^{-1}\left(\begin{array}{cc}
0 & L_{\alpha} \\
L_{\alpha} & 0
\end{array}\right) T
$$

where we have identified $L^{\infty}([-a, a]) \cong L^{\infty}([0, a])^{2}, a>0$.

Because the Mellin convolution

$$
\begin{aligned}
\hat{l}_{\alpha}(z) & :=\int_{0}^{\infty} l_{\alpha}(x) x^{z-1} d x \\
& =\frac{1}{2} \frac{\sin (\alpha z)}{\sin (\pi z)}
\end{aligned}
$$

exists for $\operatorname{Re}(z) \in(-1,1)$, we know that $L_{\alpha}$ is a bounded operator on $L^{p}\left(\left[0, \delta_{0}\right]\right), p \in[1, \infty]$, and because the function $l_{\alpha}$ is positive we get

$$
\left\|L_{\alpha}\right\|_{L^{p}} \leq \hat{l}_{\alpha}\left(\frac{1}{p}\right)
$$

But $L_{\alpha}$ is not a compact operator. For $L^{\infty}$ we get the result

$$
\left\|L_{\alpha}\right\|_{L^{\infty}} \leq \frac{1}{2} \frac{|\alpha|}{\pi}<\frac{1}{2}
$$


see $[6]$ for these properties of the Mellin convolution operator. But this does not imply $\|V\|_{L^{\infty}(\gamma)}<1 / 2$, which would be a hint that a function $w$ with (2.14) exists. Only the local parts of $V$ around each corner fulfill this condition, but the remaining compact parts are not controlled.

Now we see that the above method does not always work, but if we are able to find a partition such that the spectral radius of the corresponding matrix is smaller than $1 / 2$, we can calculate a suitable weight function $w$ which fulfills $\|\bar{W}\|_{\mathcal{C}(S), w}<1 / 2$. This leads us to the following definition.

Definition 2.3. Given a polyhedral domain $D \subset \mathbf{R}^{3}$ with boundary $S$,

$$
S=\bigcup_{j=1}^{N} S_{j}, \quad S_{j} \text { triangles, }
$$

and $n$ vertices $\mathcal{V}_{j}, j=1(1) n$. We will say that $D$ fulfills the condition $(\mathbf{F})$ if we find for every vertex $\mathcal{V}_{j}$ a partition $\mathcal{P}\left(\gamma_{j}\right)=\left\{\gamma_{i}^{(j)} \mid i=\right.$ $\left.1(1) m_{j}\right\}$ of the corresponding spherical polygon $\gamma_{j}$ such that the matrix $M\left(\mathcal{P}\left(\gamma_{j}\right)\right)$ has a spectral radius smaller than $1 / 2$. We denote by $w_{i}^{(j)}$, $i=1(1) m_{j}$, the weights around vertex $\mathcal{V}_{j}$ and by $K_{i}^{(j)}, i=1(1) m_{j}$, we denote the plane sectors around vertex $\mathcal{V}_{j}$, where the weight function has the value $w_{i}^{(j)}$. If $K_{i}^{(j)}$ and $K_{i+1}^{(j)}$ are two sectors with a common edge and if $\beta$ is the angle which is enclosed by these sectors (the smaller one) then we assume

$$
\frac{1}{2}\left(1-\frac{\beta}{\pi}\right) \max \left\{\frac{1}{w_{i}^{(j)}}, \frac{1}{w_{i+1}^{(j)}}\right\}<\frac{1}{2} .
$$

Remark. The reason for the assumption (2.18) will become clear in the proof of Lemma 3.1. This assumption is sufficient for the construction of a global weighted norm on $S$, such that the essential norm of the double layer operator is smaller than $1 / 2$ on $\mathcal{C}(S)$. If $(2.18)$ is true, we can glue together the weighted norms of different vertices. Actually condition (2.18) is too strong, see Lemma 3.1. The factor $(1-\beta / \pi) / 2$ in formula $(2.18)$ is the maximal normalized solid angle 
under which a point on $K_{i}^{(j)}$ sees $K_{i+1}^{(j)}$, and this is symmetric between $i$ and $i+1$. We would like to mention that (2.18) is fulfilled in the Král and Wendland example and for the cone $K_{A}$. For every convex cone we can choose the weight function $w(x)=1$, and (2.18) is automatically fulfilled.

3. The essential norm of the double layer potential. In this section we construct a weighted norm for $\mathcal{C}(S)$ for polyhedral domains which fulfill condition $(\mathrm{F})$, such that the essential norm of the double layer operator with respect to this norm is smaller than $1 / 2$. Here we denote the weight function on $S$ by $\Omega$ in contrast to the weight function $\omega$ on the local cones. In the next section the following theorem will be the basis for the study of the stability of the collocation method. First we define a splitting of the operator $\bar{W}$ in a noncompact part $\bar{W}_{\varepsilon}$ and a compact part $\bar{C}_{\varepsilon}$.

$$
\left(\bar{W}_{\varepsilon} u\right)(x):=\left(\frac{1}{2}-d(x)\right) u(x)+\left(W_{\varepsilon} u\right)(x),
$$

where

$$
\left(W_{\varepsilon} u\right)(x)=\int_{S} \chi_{\varepsilon}(\|x-y\|) k(x, y) u(y) d y,
$$

and

$$
\chi_{\varepsilon}(t):= \begin{cases}1, & t \leq \varepsilon \\ 0, & t>\varepsilon\end{cases}
$$

The compact operator $\bar{C}_{\varepsilon}$ is defined by

$$
\bar{C}_{\varepsilon}:=\bar{W}-\bar{W}_{\varepsilon},
$$

see, i.e., $[\mathbf{1 2}]$. Now we can formulate the main result of this section.

Theorem 3.1. Let the polyhedral domain $D$ fulfill condition (F). There exists a weight function $\Omega: S \rightarrow(0, \infty), \Omega$ has only finite many values, and an $\varepsilon_{0}>0$ such that

$$
\left\|\bar{W}_{\varepsilon}\right\|_{\mathcal{C}(S), \Omega}<\frac{1}{2}
$$


for $\varepsilon \in\left(0, \varepsilon_{0}\right)$.

Proof. First we recall that the norm $\|u\|_{\mathcal{C}(S), \Omega}$ for a continuous function $u$ is defined by

$$
\|u\|_{\mathcal{C}(S), \Omega}:=\sup _{x \in S} \frac{|u(x)|}{\Omega(x)} .
$$

Denote by $\varepsilon_{1}>0$ a constant with

$$
B_{2 \varepsilon_{1}}\left(\mathcal{V}_{i}\right) \cap B_{2 \varepsilon_{1}}\left(\mathcal{V}_{j}\right)=\varnothing, \quad i \neq j,
$$

so that the vertices are separated. For each cone $K_{j}$, corresponding to the vertex $\mathcal{V}_{j}$, we have a partition

$$
K_{j}=\bigcup_{i=1}^{m_{j}} K_{i}^{(j)},
$$

and weights $w_{i}^{(j)} \in(0,1], i=1(1) m_{j}$. Each $K_{i}^{(j)}$ is a plane sector, and we can assume (by further partition) that all $K_{i}^{(j)}$ who have an edge with $S$ in common have the same opening angle $\alpha>0$.

Now we define $\Omega$ for $x \in S$.

a. $x \in B_{\varepsilon_{1}}\left(\mathcal{V}_{j}\right) \cap K_{i}^{(j)}$. Then we define

$$
\Omega(x):=w_{i}^{(j)} .
$$

If $x$ is on the boundary of two $K_{i}^{(j)}$, we can assign one of the two weights.

b. If $x$ is not in an $\varepsilon_{1}$ neighborhood of any vertex, but has a distance smaller than $\sin (\alpha) \varepsilon_{1}$ from an edge $e$, then we assign the value corresponding to Figure 8.

c. If $x$ is not in an $\varepsilon_{1}$ neighborhood of one of the vertices and has a bigger distance than $\sin (\alpha) \varepsilon_{1}$ from any edge, we define

$$
\Omega(x):=1 .
$$

Now we define $\varepsilon_{0}:=\sin (\alpha) \varepsilon_{1}$. For $\varepsilon \in\left(0, \varepsilon_{0}\right)$ and $u \in \mathcal{C}(S)$ with $\|u\|_{\mathcal{C}(S), \Omega} \leq 1$ we will now show

$$
\left|\left(\bar{W}_{\varepsilon} u\right)(x)\right|<\frac{\Omega(x)}{2} .
$$




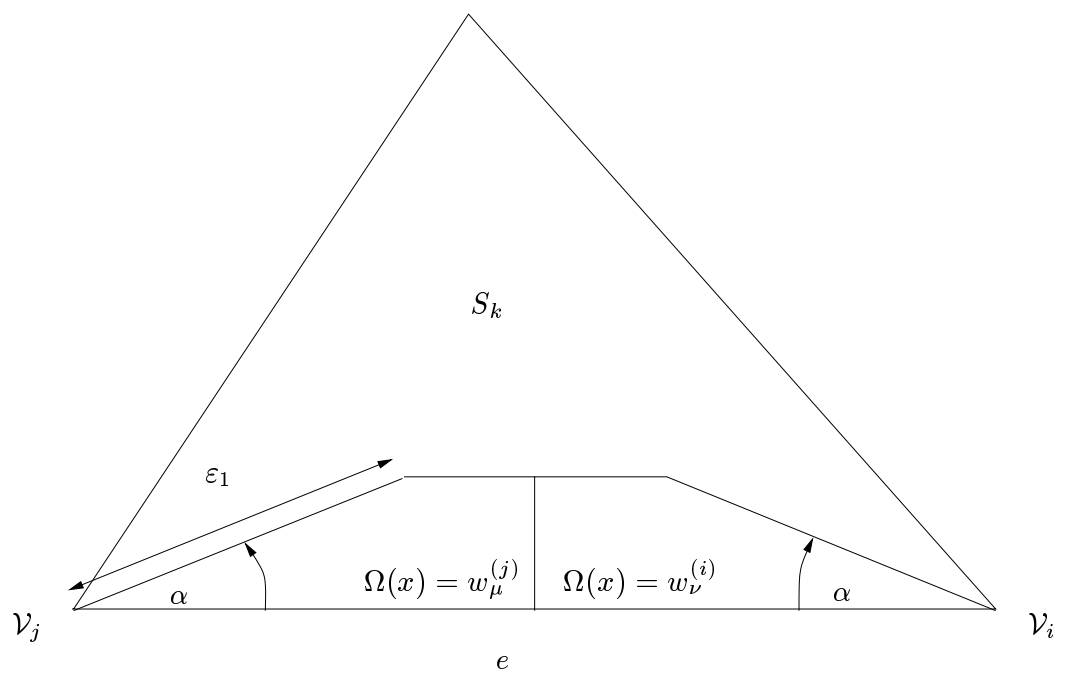

FIGURE 8. $\Omega(x)$ near the edge connecting $\mathcal{V}_{j}$ and $\mathcal{V}_{i}$.

If $x \in S$ has a distance bigger than $\varepsilon_{0}$ from any edge we get

$$
\left(\bar{W}_{\varepsilon} u\right)(x)=0
$$

which proves (3.6). If $B_{\varepsilon}(x) \subset B_{\varepsilon_{1}}\left(\mathcal{V}_{i}\right), i \in\{1, \ldots, n\}$, we get (3.6) by the construction of our weights in Lemma 2.1. Now let $x$ have a distance smaller than $\varepsilon_{0}$ from an edge $e$ and denote by $S_{1}$ and $S_{2}$ the two triangles which are adjacent to $e$. By $\beta$ we again denote the angle enclosed by $S_{1}$ and $S_{2}$, see Definition 2.3. We assume that the two vertices at the ends of $e$ are $\mathcal{V}_{1}$ and $\mathcal{V}_{2}$. Then it becomes clear that it is enough to estimate $\left(\bar{W}_{\varepsilon} u\right)(x)$ near the point $P$ of Figure 9.

In order to get (3.6) we must have

$$
\begin{aligned}
& \frac{1}{2} \frac{1}{w_{j}^{(1)}}\left(1-\frac{\beta}{\pi}\right)\left(\frac{w_{j+1}^{(1)}}{2}+\frac{w_{k+1}^{(2)}}{2}\right)<\frac{1}{2}, \\
& \frac{1}{2} \frac{1}{w_{j+1}^{(1)}}\left(1-\frac{\beta}{\pi}\right)\left(\frac{w_{j}^{(1)}}{2}+\frac{w_{k}^{(2)}}{2}\right)<\frac{1}{2}, \\
& \frac{1}{2} \frac{1}{w_{k}^{(2)}}\left(1-\frac{\beta}{\pi}\right)\left(\frac{w_{j+1}^{(1)}}{2}+\frac{w_{k+1}^{(2)}}{2}\right)<\frac{1}{2},
\end{aligned}
$$




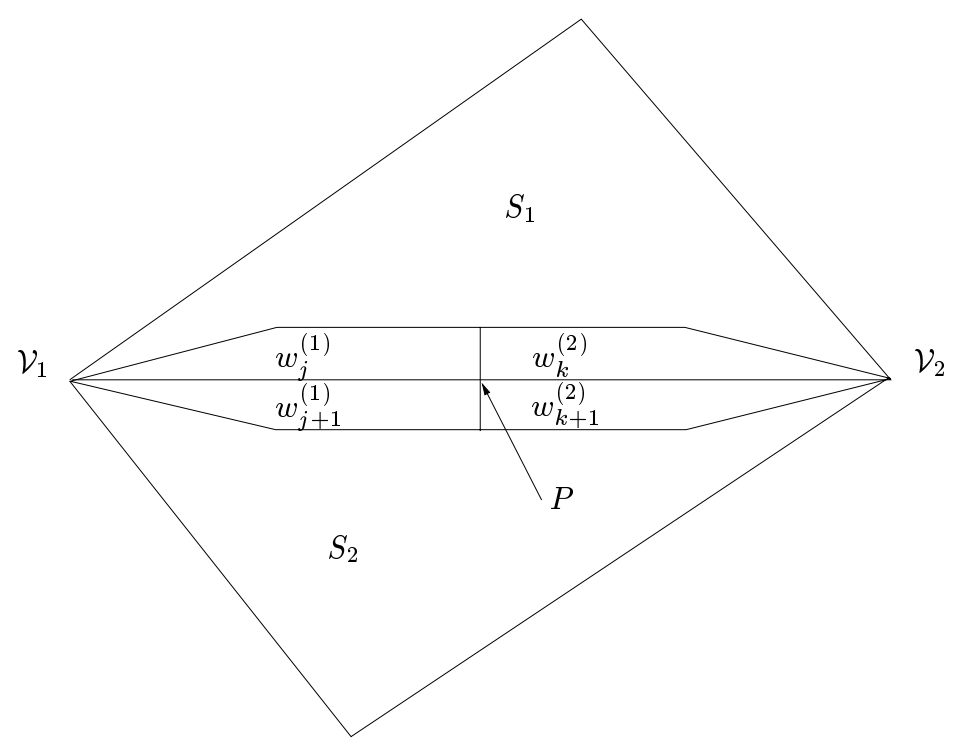

FIGURE 9. The weights along the edge $e$.

$$
\frac{1}{2} \frac{1}{w_{k+1}^{(2)}}\left(1-\frac{\beta}{\pi}\right)\left(\frac{w_{j}^{(1)}}{2}+\frac{w_{k}^{(2)}}{2}\right)<\frac{1}{2} .
$$

Because of condition $(\mathrm{F})$ we get

$$
\frac{1}{2}\left(1-\frac{\beta}{\pi}\right) \max \left\{\frac{w_{j}^{(1)}}{w_{j+1}^{(1)}}, \frac{w_{j+1}^{(1)}}{w_{j}^{(1)}}, \frac{w_{k}^{(2)}}{w_{k+1}^{(2)}}, \frac{w_{k+1}^{(2)}}{w_{k}^{(2)}}\right\}<\frac{1}{2}
$$

We further have the condition (2.18) which, together with $w_{i}^{(j)} \leq 1$, for all $i, j$ proves (3.6).

The above theorem together with the compactness of $\bar{C}_{\varepsilon}$ implies that the essential norm of $\bar{W}$ in the weighted space $\left(\mathcal{C}(S),\|\cdot\|_{\mathcal{C}(S), \Omega}\right)$ is smaller than $1 / 2$. Because the weighted norm and the usual maximum norm are equivalent, we also get 
Corollary 3.2. The above theorem implies that the operator

$$
\frac{1}{2}-\bar{W}
$$

is a Fredholm operator in $\mathcal{C}(S)$.

4. The stability of the collocation method. In this section we will investigate the stability of the collocation method for equation (1.1). Before we define exactly the collocation method, we need one more technical lemma which will be important for the stability proof.

Lemma 4.1. Let $\varepsilon \in\left(0, \varepsilon_{0}\right], u \in L^{\infty}(S)$, piecewise continuous, and define

$$
f(x):=\left(\bar{W}_{\varepsilon} u\right)(x), \quad x \in S .
$$

If $\mathcal{E}$ denotes the union of all edges of $S$ and

$$
\mathcal{E}_{\delta}:=\{x \in S \mid \operatorname{dist}(x, \mathcal{E}) \in[0, \delta)\},
$$

it follows that $f$ is Lipschitz continuous on $S \backslash \mathcal{E}_{\delta}$ for every $\delta>0$, and the Lipschitz constant $L=L\left(\|u\|_{L^{\infty}(S)}, \delta, \varepsilon\right)$ depends only on the norm of $u, \delta$ and $\varepsilon$,

$$
L \leq C_{L}(\varepsilon) \frac{\|u\|_{L^{\infty}(S)}}{\delta} .
$$

Proof. Because

$$
f(x)=\sum_{i=1}^{n} \int_{S_{i}} k(x, y) \chi_{\varepsilon}(\|x-y\|) u(y) d y
$$

it is enough to prove the assumption only for $x \in S_{j}, j \in\{1, \ldots, n\}$, and

$$
f(x)=\int_{S_{i}} k(x, y) \chi_{\varepsilon}(\|x-y\|) u(y) d y .
$$

If $S_{i}$ and $S_{j}$ have a positive distance, we get $f \in \mathcal{C}^{\infty}\left(S_{j}\right)$. So we can assume that $S_{i}$ and $S_{j}$ are adjacent and that

$$
\begin{aligned}
S_{i} & \subset\left\{\left(x_{1}, x_{2}, x_{3}\right) \mid x_{3}=0\right\} \\
S_{j} & \subset\left\{\left(x_{1}, x_{2}, x_{3}\right) \mid x_{3}>0\right\}
\end{aligned}
$$


and their common edge is, for example, a subset of $\left\{\left(0, x_{2}, 0\right) \mid x_{2} \geq 0\right\}$. If $x \in S_{j}, x_{3} \geq \varepsilon$, we get

$$
\left(\bar{W}_{\varepsilon} u\right)(x)=0
$$

so $f$ is a $\mathcal{C}^{\infty}$ function. If we denote by $\chi_{i}$ the characteristic function of $S_{i}$, we get for $x_{3} \in(0, \varepsilon]$

$$
\begin{aligned}
f(x) & =\int_{S(i)} k(x, y)\left(\chi_{\varepsilon} \chi_{i} u\right)(y) d y \\
& =\int_{B(x)} k(x, y) v(y) d y,
\end{aligned}
$$

where now $\chi_{i} u$ is again a piecewise continuous function and

$$
B(x)=\left\{\left(y_{1}, y_{2}, 0\right) \mid\left(x_{1}-y_{1}\right)^{2}+\left(x_{2}+y_{2}\right)^{2} \leq \varepsilon^{2}-x_{3}^{2}\right\}
$$

This representation already implies the continuity of $f(\cdot)$ for $x_{3}>0$, where we consider $f(\cdot)$ also as a function in $\left\{\left(x_{1}, x_{2}, x_{3}\right) \mid x_{3}>0\right\}$. We denote by

$$
c(x, \cdot):[0,2 \pi] \longrightarrow \mathbf{R}^{3},
$$

a parametrization of the boundary of $B(x)$ given by

$$
c(x, s):=\left(\begin{array}{l}
x_{1} \\
x_{2}
\end{array}\right)+\sqrt{\varepsilon^{2}-x_{3}^{2}}\left(\begin{array}{c}
\cos (s) \\
\sin (s)
\end{array}\right) .
$$

One can compute that for $\nu \in \mathbf{R}^{3}$, the directional derivative $(\partial f / \partial \nu)(x), x_{3} \in(0, \varepsilon)$, is given by

$$
\begin{aligned}
\frac{\partial f}{\partial \nu}(x)= & \int_{B(x)} \frac{\partial k}{\partial \nu_{x}}(x, y) v(y) d y \\
& +\int_{0}^{2 \pi} k(x, c(x, s)) v(c(x, s)) \operatorname{det}\left(\left.\frac{\partial c(x+\tau \nu, \sigma)}{\partial(\tau, \sigma)}\right|_{\tau=0}\right) d \sigma
\end{aligned}
$$

Remembering

$$
k(x, c(x, s))=\frac{1}{4 \pi} \frac{x_{3}}{\sqrt{\varepsilon^{2}-x_{3}^{2}}}
$$


we see that the second integral is a smooth function near $x_{3}=0$ and only the first integral has to be considered near $x_{3}=0$. We have (if $\left.n(y)=(0,0,-1)^{T}, y \in S_{i}\right)$

$$
\frac{\partial k}{\partial \nu_{x}}=-\frac{1}{4 \pi}\left(\frac{\nu_{3}}{\|x-y\|^{3}}-3 x_{3} \frac{\nu \cdot(x-y)}{\|x-y\|^{5}}\right) .
$$

We estimate the first integral by (assume $\|\nu\|=1$ )

$$
\begin{aligned}
& \int_{B(x)}\left|\frac{\partial k}{\partial \nu_{x}}(x, y) v(y)\right| d y \\
& \leq \frac{\|v\|_{L^{\infty}}}{4 \pi} \int_{0}^{\infty} \int_{0}^{2 \pi}\left[\frac{1}{\left(x_{3}^{2}+r^{2}\right)^{3 / 2}}\right. \\
& \left.\quad+3 x_{3}\left(\frac{\left|\nu_{1}\right| r|\cos (\varphi)|+\left|\nu_{2}\right| r|\sin (\varphi)|+\left|\nu_{3}\right| x_{3}}{\left(x_{3}^{2}+r^{2}\right)^{5 / 2}}\right)\right] r d \varphi d r \\
& \leq 3\|v\|_{L^{\infty}} \int_{0}^{\infty} \frac{1}{x_{3}^{2}} \frac{r / x_{3}}{\left(1+\left(r / x_{3}\right)^{2}\right)^{3 / 2}}+\frac{1}{x_{3}^{2}} \frac{\left(r / x_{3}\right)^{2}}{\left(1+\left(r / x_{3}\right)^{2}\right)^{5 / 2}} \\
& =3 \frac{\|v\|_{L^{\infty}}}{x_{3}} \int_{0}^{\infty} \frac{s}{\left(1+s^{2}\right)^{3 / 2}}+\frac{s^{2}}{\left(1+s^{2}\right)^{5 / 2}}+\frac{s / x_{3}}{\left(1+s^{2}\right)^{5 / 2}} d s \\
& \leq\|v\|_{L^{\infty}} C_{1} \frac{1}{\operatorname{dist}\left(x, S_{i}\right)} .
\end{aligned}
$$

where the constant $C_{1}$ also depends on the angle between $S_{i}$ and $S_{j}$, but we only have finite many triangles and angles in $S$. So we get that $f(\cdot)$ is continuous in $x_{3}>0$, constant to zero in $x_{3}>\varepsilon$, and $f$ is differentiable in $x_{3} \in(0, \varepsilon)$ with the bound for the gradient given in the last inequality. This proves our lemma.

Now we define a collocation method for the solution of (1.1) with piecewise continuous trial functions. We assume that we have a sequence of grids $G_{j}, j \in \mathbf{N}$,

$$
\begin{aligned}
G_{j} & =\left\{\Delta_{1}^{(j)}, \ldots, \Delta_{k_{j}}^{(j)}\right\}, \\
S & =\bigcup_{i=1}^{k_{j}} \Delta_{i}^{(j)}, \\
\dot{\Delta}_{i}^{(j)} \cap \dot{\Delta}_{k}^{(j)} & =\varnothing, \quad i \neq k .
\end{aligned}
$$


Define further

$$
d_{j, i}:=\operatorname{diam}\left(\Delta_{i}^{(j)}\right), \quad \bar{d}_{j}:=\max _{i=1}^{k_{j}}\left\{d_{j, i}\right\} .
$$

The $G_{j}$ may be, for example, some graded grids in order to get high convergence rates, but we will not assume any special properties besides

$$
\lim _{j \rightarrow \infty} \bar{d}_{j}=0 .
$$

For the reference triangle $\Delta_{\text {Ref }}:=\left\{(x, y) \in \mathbf{R}^{2} \mid 0 \leq x, y ; x+y \leq 1\right\}$ we consider two different collocation projectors for continuous functions $f$ on $\Delta_{\text {Ref }}$

$$
\left(P_{0} f\right)(x):=f\left(\frac{1}{3}, \frac{1}{3}\right), \quad x \in \Delta_{\mathrm{Ref}}
$$

and

$$
\left(P_{1} f\right)(x):=\sum_{i, j \geq 0, i+j \leq r} c_{i, j} x_{1}^{i} x_{2}^{j}, \quad x \in \Delta_{\text {Ref }}
$$

such that

$$
\left(P_{1} f\right)\left(\xi_{i, j}\right)=f\left(\xi_{i, j}\right), \quad 0 \leq i, j ; i+j \leq r ; \xi_{i, j} \in \dot{\Delta}_{\text {Ref }} .
$$

So the second interpolation formula has $f_{r}=(r+1)(r+2) / 2$ coefficients, degree $r$, and we get

$$
\left\|\left(P_{1} f\right)-f\right\|_{\mathcal{C}\left(\Delta_{\text {Ref }}\right)} \leq\left\|P_{1}\right\| \sup _{x, y \in \Delta_{\text {Ref }}}\|f(x)-f(y)\|,
$$

see [2, Lemma 5.1.1], and it is clear that the $\xi_{i, j}$ could not be chosen arbitrarily. We can also find a Lagrange basis for the interpolation

$$
\left(P_{1} f\right)(x)=\sum_{i, j \geq 0, i+j \leq r} f\left(\xi_{i, j}\right) l_{i, j}(x) .
$$

For the triangulation we will further assume some regularity; this means if

$$
T_{i}^{(j)}: \Delta_{\operatorname{Ref}} \stackrel{1: 1}{\longrightarrow} \Delta_{i}^{(j)}
$$


are the parameterizations of the triangles $\Delta_{i}^{(j)}$ then

$$
d_{j, i}\left\|\left[T_{i}^{(j)}\right]^{-1}\right\| \leq C_{T}, \quad \forall i, j, C_{T} \text { a constant. }
$$

Now we introduce a parameter $\varepsilon^{*}$ to define the trial space for the collocation method. First we split the triangles in $G_{j}$ :

$$
G_{j}=G_{j, 0}\left(\varepsilon^{*}\right) \cup G_{j, 1}\left(\varepsilon^{*}\right)
$$

where

$$
\Delta_{i}^{(j)} \in \begin{cases}G_{j, 1}\left(\varepsilon^{*}\right), & \text { if } \frac{d_{j, i}}{\operatorname{dist}\left(\Delta_{i}^{(j)}, \mathcal{E}\right)} \leq \varepsilon^{*} \\ G_{j, 0}\left(\varepsilon^{*}\right), & \text { otherwise. }\end{cases}
$$

The last assumption on our triangulation is

$$
\left.\Omega\right|_{\Delta_{i}^{(j)}}=\text { const }, \quad \forall \Delta_{i}^{(j)} \in G_{j, 0} .
$$

This last assumption can be achieved by starting with a triangulation $G_{1}$ which already respects the condition (4.13) and by further refinement. The trial space for the collocation method is given by

$$
\begin{aligned}
\Pi_{j}\left(\varepsilon^{*}\right) & :=\left\{\chi_{\Delta_{i}^{(j)}} \mid i \in G_{j, 0}\left(\varepsilon^{*}\right)\right\} \\
& \cup\left\{\chi_{\Delta_{i}^{(j)}} l_{k, l}\left(\left[T_{i}^{(j)}\right]^{-1} x\right) \mid i \in G_{j, 1}\left(\varepsilon^{*}\right), 0 \leq k, l ; k+l \leq r\right\},
\end{aligned}
$$

where $\chi_{M}$ denotes the characteristic function of a set $M$. The definition of $\Pi_{j}\left(\varepsilon^{*}\right)$ looks very complicated but it just means the following: Given a sequence of triangulations $\left(G_{j}\right)_{j \in \mathbf{N}}$, a number $r \in \mathbf{N}_{0}$ and a positive number $\varepsilon^{*}$, we use piecewise constant interpolation functions on all triangles of $G_{j}$ which are relatively close to the edges, see (4.12), and polynomials of maximal degree $r$ on all other triangles. The collocation points are defined by

$$
\begin{aligned}
\Phi_{j}\left(\varepsilon^{*}\right) & :=\left\{\left[T_{i}^{(j)}\right]\left(\frac{1}{3}, \frac{1}{3}\right) \mid i \in G_{j, 0}\left(\varepsilon^{*}\right)\right\} \\
& \cup\left\{\left[T_{i}^{(j)}\right]\left(\xi_{k, l}\right) \mid i \in G_{j, 1}\left(\varepsilon^{*}\right), 0 \leq k, l ; k+l \leq r\right\},
\end{aligned}
$$


and the interpolation projector

$$
\left\{\begin{aligned}
P_{j, \varepsilon^{*}}: L^{\infty}(S) & \rightarrow \Pi_{j}\left(\varepsilon^{*}\right) \\
\left(P_{j, \varepsilon^{*}} u\right)(x) & =u(x), \quad \forall x \in \Phi_{j}\left(\varepsilon^{*}\right), u \in \mathcal{C}(S)
\end{aligned}\right.
$$

See $[\mathbf{3}]$ for the extension of the interpolation projector to $L^{\infty}(S)$. The collocation method is given by: Find $u_{j} \in \operatorname{span}\left(\Pi_{j}\right)\left(\varepsilon^{*}\right)$ such that

$$
\left(\frac{1}{2} I-\bar{W}\right) u_{j}(x)=f(x), \quad \forall x \in \Phi_{j}\left(\varepsilon^{*}\right)
$$

After all these definitions we can formulate our stability result.

Theorem 4.2. Let $D \subset \mathbf{R}^{3}$ be a polyhedral domain with boundary $S$ which fulfills condition (F). Given a sequence $G_{j}, j \in \mathbf{N}$, of triangulations of $S$ with (4.3), (4.10) and (4.13) and the interpolation projectors $P_{0}$ and $P_{1}$. Then there is an $\bar{\varepsilon}^{*}$, such that for all $\varepsilon^{*} \in\left(0, \bar{\varepsilon}^{*}\right]$ and sufficiently large $j$ we get

$$
\begin{gathered}
\left\|P_{j, \varepsilon^{*}}\left(\frac{1}{2} I-\bar{W}\right) u\right\|_{L^{\infty}(S), \Omega} \geq c_{S}\|u\|_{L^{\infty}(S), \Omega}, \\
\forall u \in \operatorname{span}\left(\Pi_{j}\left(\varepsilon^{*}\right)\right) .
\end{gathered}
$$

Proof. Choosing $\varepsilon>0$ sufficiently small, we know that there exists a function $\Omega: S \rightarrow \mathbf{R}$, such that

$$
\begin{aligned}
\left\|\bar{W}_{\varepsilon}\right\|_{L^{\infty}(S), \Omega} & \leq q \leq \frac{1}{2} \\
0<c_{\Omega} & \leq \Omega(x) \leq C_{\Omega} .
\end{aligned}
$$

The operator $I / 2-\bar{W}$ is invertible, see [14], and the operator $\bar{C}_{\varepsilon}$ is compact. By [8] we also know that we have to show (4.18) only for $I / 2-\bar{W}_{\varepsilon}$ and then (4.18) follows for sufficiently large $j$. Now choose 
an $\varepsilon^{*}>0$ and consider the interpolation error on a triangle in $G_{j, 1}\left(\varepsilon^{*}\right)$

$$
\begin{aligned}
& \left|\left[P_{j, \varepsilon^{*}}\left(\bar{W}_{\varepsilon} u\right)\right](x)-\left(\bar{W}_{\varepsilon} u\right)(x)\right| \\
& \quad=\quad\left|\left[P_{1}\left(\left(\bar{W}_{\varepsilon} u\right) \circ\left[T_{i}^{(j)}\right]^{-1}\right)\right](x)-\left(\left(\bar{W}_{\varepsilon} u\right) \circ\left[T_{i}^{(j)}\right]^{-1}\right)(x)\right| \\
& \quad \stackrel{(4.7)}{\leq}\left\|P_{1}\right\| \sup _{x, y \in \Delta_{\text {Ref }}}\left|\left(\left(\bar{W}_{\varepsilon} u\right) \circ\left[T_{i}^{(j)}\right]^{-1}\right)(x)-\left(\left(\bar{W}_{\varepsilon} u\right) \circ\left[T_{i}^{(j)}\right]^{-1}\right)(y)\right| \\
& \quad\left\|P_{1}\right\| \sup _{x, y \in \Delta_{i}^{(j)}}\left|\left(\bar{W}_{\varepsilon} u\right)(x)-\left(\bar{W}_{\varepsilon} u\right)(y)\right| \\
& \quad \underset{\quad \text { Lemma }}{\leq}\left\|P_{1}\right\|\|u\|_{L^{\infty}} C_{L}(\varepsilon) \frac{d_{j, i}}{\operatorname{dist}\left(\Delta_{i}^{(j)}, \mathcal{E}\right)} \\
& \quad(4.12) \\
& \quad\left\|P_{1}\right\| C_{T} C_{L}(\varepsilon) \varepsilon^{*}\|u\|_{L^{\infty}} .
\end{aligned}
$$

Define $q^{\prime}:=(1 / 2+q) / 2<1 / 2$. Then we get for $x \in \Delta_{i}^{(j)} \in G_{j, 1}$ and $\|u\|_{L^{\infty}(S), \Omega}=1$

$$
\begin{aligned}
& \frac{\left|\left[P_{j, \varepsilon^{*}}\left(\bar{W}_{\varepsilon} u\right)\right](x)\right|}{\Omega(x)} \leq \frac{\left|\left[P_{j, \varepsilon^{*}}\left(\bar{W}_{\varepsilon} u\right)\right](x)-\left(\bar{W}_{\varepsilon} u\right)(x)\right|+\left|\left(\bar{W}_{\varepsilon} u\right)(x)\right|}{\Omega(x)} \\
& \stackrel{\text { Theorem } 3.1}{\leq} \frac{\left\|P_{1}\right\|}{c_{\Omega}} C_{T} C_{L}(\varepsilon) C_{\Omega} \varepsilon^{*}+q \\
& \leq q^{\prime}
\end{aligned}
$$

if $\varepsilon^{*} \in\left(0, \bar{\varepsilon}^{*}\right)$, if $\bar{\varepsilon}^{*}>0$ is sufficiently small. If $x \in \Delta_{i}^{(j)} \subset G_{j, 0}$, with collocation point $\xi$ we get

$$
\begin{aligned}
\frac{\left|\left[P_{j, \varepsilon^{*}}\left(\bar{W}_{\varepsilon} u\right)\right](x)\right|}{\Omega(x)} & =\frac{\left|\left(\bar{W}_{\varepsilon} u\right)(\xi)\right|}{\Omega(\xi)} \\
& \leq q<q^{\prime} .
\end{aligned}
$$

But the last two inequalities show

$$
\begin{gathered}
\sup _{x \in S} \frac{\left|u(x) / 2-\left[P_{j, \varepsilon^{*}}\left(\bar{W}_{\varepsilon} u\right)\right](x)\right|}{\Omega(x)} \geq \frac{1}{2}-q^{\prime}, \\
u \in \operatorname{span}\left(\Pi_{j}\left(\varepsilon^{*}\right)\right),\|u\|_{L^{\infty}, \Omega}=1 .
\end{gathered}
$$

This proves our theorem and the stability of the collocation method.

口 
Remark. It is clear that the modification of the collocation method near the edges can be omitted if the collocation projector has norm 1. This means that the panel method, see [14], is stable without any modification.

Acknowledgments. The author would like to thank Professor K. Atkinson, University of Iowa, and Professor Dr. H. Mülthei, Johannes Gutenberg-Universität Mainz, who made it possible for him to spend half a year at the University of Iowa, Iowa City. He would like to especially thank Professor Atkinson for his kind assistance during this half year, and for many suggestions and explanations concerning integral equations.

\section{REFERENCES}

1. T.S. Angell, R.E. Kleinman and J. Král, Layer potentials on boundaries with corners and edges, Casopis Pěst. Mat. 113 (1986), 387-403.

2. K. Atkinson, The numerical solution of integral equations of the second kind, Cambridge University Press, Cambridge, 1997.

3. K. Atkinson, I. Graham and I. Sloan, Piecewise continuous collocation for integral equations, SIAM J. Numer. Anal. 20 (1983), 172-186.

4. P.K. Banerjee, The boundary element method in engineering, McGraw-Hill Internat., UK, 1994.

5. G. Chandler and I. Graham, Product integration- Collocation methods for non-compact integral operator equations, Math. Comp. 50 (1988), 125-138.

6. J. Elschner, On Spline approximation for a class of non-compact integral equations, Math. Nachr. 146 (1990), 271-321.

7. — The double layer potential operator over polyhedral domains I: Solvability in weighted Sobolev spaces, Appl. Anal. 45 (1992), 117-134.

8. I.Z. Gochberg and I.A. Feldman, Faltungsgleichungen und Projektionsverfahren zu ihrer Lösung, Birkhäuser Verlag, Basel, 1974.

9. N.V. Grachev, Representations and estimates for inverse operators of the potential theory integral equations in a polyhedron, in Potential theory (M. Kishi, ed.), Walter de Gruyter, Berlin, 1991.

10. N.V. Grachev and V.G. Maz'ya, Solvability of a boundary integral equation on a polyhedron, Department of Mathematics, Linköping University, Preprint LITHMAT-R91-50, 1992.

11. J. Král, Integral operators in potential theory, Lecture Notes in Math. 823, Springer-Verlag, Berlin, New York, 1980.

12. J. Král and W. Wendland, Some examples concerning applicability of the Fredholm-Radon method in potential theory, Aplik. Math. 31 (1986), 293-308. 
13. V.G. Maz'ya, Boundary integral equations, in Analysis IV, Encyclopaedia of Math. Sci., Vol. 27, Springer Verlag, Berlin, New York, 1991.

14. A. Rathsfeld, The invertibility of the double layer potential operator in the space of continuous functions defined on a polyhedron. The panel method, Appl. Anal. 45 (1992), 135-177.

15. M.A. Woodbury, Characteristic roots of input-output matrices, in Economic activity analysis (O. Morgenstern, ed.), John Wiley \& Sons, Inc., New York, London, 1954.

Fachbereich Mathematik, Johannes-Gutenberg Universität Mainz, SaArstr. 21, 55099 Mainz, Germany

E-mail address: hansen@mathematik.uni-mainz.de 